\title{
Análise empírica da volatilidade do etanol: aplicação de modelos ARCH nos preços à vista e futuros no período 2011-2015
}

\author{
RAMON RODRIGUES DOS SANTOS*
}

\begin{abstract}
RESUMO
Esta pesquisa teve a finalidade de analisar a dinâmica e a transmissão da volatilidade dos preços à vista e futuros do etanol pelos modelos $\mathrm{ARCH}, \mathrm{GARCH}, \mathrm{EGARCH}$ e TARCH. A amostra utilizada nesta pesquisa é composta pelas cotações diárias do etanol hidratado spot negociado na base de Paulínia (SP), disponibilizadas pelo CEPEA/ ESALQ e das cotações futuro na CBOT (Chicago Board of Trade) Denatured Fuel Ethanol Futures), no período de 03/10/2011 a 27/11/2015, em um total de 2.032 observações. Dentre os modelos analisados, destaca-se que os modelos EGARCH $(2,2)$ para o spot e EGARCH $(1,2)$ para o futuro são os que possuem o melhor ajustamento da série, considerado os Critérios de Informação de Akaike (AIC) e de Schwarz (SC), demonstrando um efeito assimetria principalmente na série de retornos futuros do etanol, e mostrando que os impactos resultantes dos choques positivos e negativos foram diferenciados na volatilidade, dentro do período analisado.
\end{abstract}

Palavras-Chave: Etanol. Volatilidade. Modelos ARCH.

\begin{abstract}
This research had the purpose of analyzing the dynamics and the transmission of the volatility of the spot and future prices of ethanol by the ARCH, GARCH, EGARCH and TARCH models. The sample used in this research is composed of daily quotations of hydrous ethanol spot traded at the Paulínia (SP) base, made available by CEPEA/ ESALQ and future quotations at the CBOT (Chicago Board of Trade) Denatured Fuel Ethanol Futures) in the period of 10/03/2011 to $11 / 27 / 2015$, for a total of $2,032 \mathrm{ob}-$ servations. Among the analyzed models, the EGARCH (2.2) models for the spot and $\operatorname{EGARCH}(1,2)$ for the future are the ones that have the best fit of the series, considered the Akaike Information Criteria (AIC) and Schwarz (SC), showing an asymmetry effect mainly in the series of future ethanol returns and showing that the impacts resulting from the positive and negative shocks were differentiated in volatility within the analyzed period.
\end{abstract}

Keywords: Ethanol. Volatility. ARCH Models.

* Universidade Federal de Pernambuco. Mestrado em Administração pelo Programa de Pós-Graduação em Administração (PPGA) da Universidade Federal da Paraíba. Professor da Universidade Federal de Pernambuco. E-mail: ramonrdgs@gmail.com . 


\section{INTRODUÇÃo}

O conceito de risco pode ser considerado um dos pilares da gestão financeira, uma vez que as estratégias de tomadores de decisão são desenvolvidas geralmente em um ambiente de incerteza em relação aos seus resultados. Neste aspecto, o conhecimento e a análise dos fatores que podem gerar estas incertezas, apresentam-se como pontos fundamentais para a otimização dos processos decisórios dos referidos agentes.

Constantemente, agentes de mercado buscam uma estrutura ótima para a minimização das incertezas e da alocação de seus recursos, equilibrando as suas relações de risco com o retorno e, por consequência, uma utilidade esperada (COPELAND; WESTON; SHASTRI, 2005). Por sua vez, produtores buscam, para garantir a sua produção desde o plantio até a colheita, assegurar um preço que seja suficiente para que, além de cobrir os custos inerentes à atividade, possuam uma margem de lucro que permita a continuidade da sua produção.

Em linhas gerais, estes produtores estão expostos a três tipos de riscos, a saber: clima, crédito e preço, e que, para cada um destes riscos, apresentam-se ferramentas capazes de gerar eficiência para os respectivos agentes (SOUZA, 2013). Em relação ao risco de preços, a aplicação em instrumentos derivativos, por exemplo, foi impulsionada por esta necessidade de proteção (hedge) das variações de preços, proporcionando uma transferência deste risco para uma contraparte, e melhor gerenciamento da incerteza por meio dos mercados financeiros. (BM\&FBOVESPA; CVM, 2015).

Em geral, o preço de uma commodity deve ser baseado em fundamentos microeconômicos (das curvas de oferta e demanda). Entretanto, os desdobramentos macroeconômicos existentes em cada país e no exterior, associados às incertezas e negociações no mercado, são fundamentos também considerados para a formação deste preço. Quanto maior o número de informações, os preços à vista (também denominados como preços spot) tendem a mudar mais rápido do que quando existe tranquilidade nos mercados, tornando-se mais voláteis, e esta volatilidade desempenha um papel importante no gerenciamento de risco e na dinâmica dos preços futuros (LIEBERG, 2014).

Além disso, incertezas relacionadas às mudanças do clima, da segurança energética e alimentar, associadas à redução dos combustíveis fósseis, provocaram e aumentaram a volatilidade dos preços das commodities agropecuárias, e, sobretudo, àquelas relacionadas as energias renováveis e aos biocombustíveis, que, por sua vez, estão no topo da agenda das políticas energéticas de diversos países. Para a Agência Nacional do Petróleo, Gás Natural e Biocombustíveis (ANP, 2015), a matriz energética mundial ainda é muito dependente dos combustíveis fósseis, um bem não renovável, logo, quaisquer movimentos em direção aos biocombustíveis tendem a "movimentar" os preços.

A demanda de etanol é dividida entre a demanda de etanol anidro e hidratado, sendo este utilizado como combustível puro e o anidro, misturado à gasolina. A valorização do álcool anidro no mercado interno intensificou-se com a Segunda Guerra Mundial, devido à escassez dos derivados de petróleos, e mais especificamente com o "choque do petróleo" em 1973 (VIAN; LIMA; ABDO, 2007). 
Dado o contexto de que os preços à vista do etanol negociados no Brasil, possuem um comportamento sazonal, uma vez que durante os meses de dezembro a março, na entressafra, as cotações tendem a elevar-se, caindo até o final do ano (MORAES; BACCHI, 2014), propõem-se alternativas de gerenciamento de risco ao estimar a volatilidade destas commodities. Ademais, somado ao fato de que o contrato futuro de etanol negociado na CBOT é mais líquido, quando comparado com o contrato futuro de etanol hidratado negociado no Brasil (TONIN; PARRÉ; TONIN, 2014) propõe-se a utilização destes contratos como uma fonte para negociação e internacionalização da commodity brasileira.

Logo, o propósito deste artigo é contribuir para os estudos sobre a dinâmica e a transmissão de volatilidade dos retornos dos preços à vista e futuros do etanol hidratado por meio das extensões dos modelos ARCH. Partindo-se do pressuposto de que a mensuração da dinâmica da volatilidade dos preços à vista e futuros do etanol pode ser feita através dos modelos heterocedásticos de variância condicional (modelos ARCH) e de suas respectivas extensões, GARCH, EGARCH e TARCH, os gestores e investidores do etanol poderão realizar estimativas de prováveis retornos que envolvam os seus investimentos, buscando auxiliar, assim, as decisões de produção da commodity.

\section{Modelos ARCH}

A dinâmica dos preços agropecuários e das séries financeiras fazem com que suas cotações oscilem consideravelmente, de um período para o outro, o que induz a uma heterogeneidade na variância do erro de previsão e a possível existência de autocorrelação destes erros de previsão. Neste aspecto, os erros de uma determinada série dependeriam dos desvios da regressão. Com isso, seria mais realista e desejável a existência de uma razão ótima de hedge dinâmica, uma vez que as perdas no mercado podem ser muitas quando a tomada de decisão é feita com base em modelos não adequados (SOUZA, 2013).

Neste aspecto, o modelo ARCH (Autoregressive Conditional Heteroscedasticity), proposto por Engle (1982) e o modelo GARCH, desenvolvido por Bollerslev (1986), são os dois métodos empíricos mais utilizados para modelar a volatilidade na literatura financeira. Em linhas gerais, o modelo baseia-se na estimação da variância condicional da série, em vez de considerar esta variância constante ao longo do tempo, fato este presente nos modelos estáticos.

Para Engle (1982), os erros de previsão existentes tendiam a ocorrer na forma de clusters, o que denota a presença de heterocedasticidade na qual a variância dos erros dependia do erro anterior, ou seja, o retorno de hoje dependeria do retorno observado no passado. Com isso, o modelo tem a finalidade de capturar esta heterocedasticidade condicional dos retornos financeiros, partindo do pressuposto de que este retorno não seria correlacionado serialmente, e com isso, sendo possível modelar simultaneamente a média e a variância de uma série, ora utilizando o conceito de variância condicional, modelada por um termo autorregressivo (AR).

Com isso, a variância do termo estocástico está correlacionada com a variável explicativa e, com isso, a variância, dita condicional, depende da observação do próprio erro 
(BUENO, 2011). Em um modelo ARCH (1), por exemplo, a variância do erro dependerá de uma constante, mais o termo . Neste contexto, o modelo ARCH é denotado por:

$$
\sigma_{t}=\alpha_{0}+\alpha_{i} \varepsilon_{t-i}^{L}+\cdots+\alpha_{p} \varepsilon_{t-p}^{L}
$$

Em que: $\sigma_{t}$ é a volatilidade condicional, $\alpha_{0}$ é a constante, $\alpha_{i}$, o coeficiente de reação da volatilidade no período $i$ e $\alpha_{p} \varepsilon_{t-p}^{2}$ os efeitos em $p$ períodos anteriores, no qual $\varepsilon_{t}$ é ruído branco.

Com uma extensão dos modelos ARCH, Bollerslev (1986) buscou suprir a lacuna nos modelos econométricos em séries temporais, que na época, além de enfatizar apenas o primeiro momento condicional, apresentava alguns fatores limitadores como tratar retornos positivos e negativos de forma semelhante, uma vez que o modelo aplica os quadrados dos retornos. Além disso, uma vez que séries financeiras tendem a não ter uma distribuição normal padrão, os modelos GARCH teriam a capacidade de modelar este fato estilizado, além de ser uma alternativa mais parcimoniosa, com um menor número de parâmetros e mais abrangente, por contemplar a variância condicional dos estimadores (OLIVEIRA NETO; GARCIA; MACHADO, 2012).

Para a estimação dos modelos GARCH utiliza-se geralmente o método de máxima verossimilhança, partindo do pressuposto de uma distribuição normal, ou na presença de leptocurtose, uma distribuição t-student ou distribuição erro generalizado. No entanto, mesmo na falta de normalidade, pode-se utilizar a distribuição normal, como uma estimação de quase-máxima-verossimilhança (BUENO, 2011).

Um modelo GARCH $(p, q)$ pode ser definido por:

$$
\sigma_{t}^{2}=\alpha_{0}+\sum_{i=1}^{p} \alpha_{i} \varepsilon_{t-i}^{2}+\sum_{i=1}^{q} \beta_{j} \sigma_{t-i}^{2}
$$

Em que: $\sigma_{t}$ é a volatilidade condicional, $\alpha_{0}$ a constante, $\alpha_{i}$ o coeficiente de reação da volatilidade, $\beta_{j}$ o coeficiente de persistência da volatilidade (o efeito GARCH), $\sigma_{t-i}^{2}$ a variância condicional no período $i$ e $\varepsilon_{t-i}^{2}$ os efeitos de em $i$ períodos anteriores.

Em sua forma mais simples, por exemplo, um $\operatorname{GARCH}(1,1)$ teria a seguinte forma funcional:

$$
\sigma_{t}^{2}=\alpha_{0}+\alpha_{1} \varepsilon_{t-1}^{2}+\beta_{1} \sigma_{t-1}^{2}
$$


Com isso, a função de variância condicional inclui as variâncias passadas $\left(\beta_{j} \sigma_{t-j}^{2}\right)$, , isto é, dos quadrados dos erros anteriores e também de sua própria variância. Neste contexto, o estudo das volatilidades em um contexto multivariado possui importantes aplicações financeiras no gerenciamento de risco, incorporando taxas dinâmicas de hedge como um instrumento refletiria as oscilações inerentes às commodities, comparado aos modelos dos quais a variância e covariância são constantes.

Neste aspecto, outras extensões ou especificações dos modelos GARCH foram desenvolvidos com a finalidade de permitir uma utilização mínima de parâmetros, e com a imposição de restrições que assegurem uma definição positiva para quaisquer valores destes parâmetros (OLIVEIRA NETO; GARCIA; MACHADO, 2012).

No modelo EGARCH (Exponential GARCH), por exemplo, desenvolvido por Nelson (1991), busca-se analisar a assimetria da volatilidade nos retornos spot da commodity, os quais possuem uma tendência exponencial e não quadrática. Em seu modelo mais simples, o EGARCH (1,1), o logaritmo da variância condicional pode ser definido como:

$$
\ln \left(\sigma_{t}^{2}\right)=\alpha_{0}+\beta_{1} \ln \left(\sigma_{t-1}^{2}\right)+\alpha_{1}\left|\frac{\varepsilon_{t-1}}{\sigma_{t-1}}\right|+\gamma_{1} \frac{\varepsilon_{t-1}}{\sigma_{t-1}}
$$

Em que: $\ln \left(\sigma_{t}^{2}\right)$ é o logaritmo neperiano da variância condicional, $\alpha_{0}$ a constante, $\beta_{1}$ o coeficiente da persistência da volatilidade, $\alpha_{1}\left|\frac{\varepsilon_{t-1}}{\sigma_{t-1}}\right|$ o termo de reação da volatilidade e o $\gamma_{1} \frac{\varepsilon_{t-1}}{\sigma_{t-1}}$ o parâmetro que capta o efeito da assimetria da volatilidade na série analisada.

De acordo com Nelson (1991), a volatilidade é maior em resposta às notícias negativas, uma vez que os retornos se apresentam abaixo do esperado pelo investidor, em detrimento às boas notícias, que influenciam os altos retornos. Com isso, caso o $\gamma=0$, indica-se a ausência de assimetria na volatilidade. Caso $\gamma \neq 0$, infere-se sobre a presença de choques positivos ou negativos na volatilidade da série.

O último modelo estimado, o TARCH, desenvolvido por Zakoian (1994), buscou capturar o efeito alavancagem nas séries de retornos, acrescido ao efeito assimetria do modelo EGARCH, partindo do pressuposto que informações, sejam estas positivas ou negativas, quando incorporadas ao mercado, originam diferentes impactos sobre a volatilidade, seja pelo sinal do choque quanto pela sua proporção. O modelo mais simples, TARCH $(1,1)$ possui a seguinte forma funcional:

$$
\sigma_{t}^{2}=\alpha_{0}+\alpha_{1} \varepsilon_{t-1}^{2}+\beta_{1} \sigma_{t-1}^{2}+\gamma_{1} d_{t-1} \varepsilon_{t-1}^{2}
$$

Em que: $\sigma_{t}^{2}$ é a volatilidade condicional, $\alpha_{0}$ o termo constante, $\alpha_{1}$ é o coeficiente de reação da volatilidade, $\varepsilon_{t-1}^{2}$ é o termo de erro ao quadrado no período $\mathrm{t}-1, \beta_{1}$ é o constante de persistência da volatilidade, $\sigma_{t-1}^{2}$ é a variância da volatilidade no período t$1, \gamma$ é o efeito assimetria e $d_{t-1}$ é a variável binária que capta o efeito alavancagem. Se $\gamma>0$, identifica-se a presença do efeito alavancagem, nos quais choques negativos originam um impacto maior na volatilidade da série do que em choques positivos. 


\section{Procedimentos Metodológicos}

\subsection{Amostra da Pesquisa}

A amostra utilizada nesta pesquisa é composta pelos dados diários do etanol hidratado à vista e futuros, no período de 03/10/2011 a 27/11/2015, em um total de 1.028 observações (à vista) e 1.047 observações futuro diárias, totalizando 2.075 observações. A série de preços spot foi a disponibilizada pelo Centro de Estudos Avançados em Economia Aplicada - ESALQ/USP (base Paulínia (SP) ESALQ) e as cotações diárias futuras do etanol, na Chicago Mercantile Exchange Futures Data.

Tabela 1 - Amostra da Pesquisa

\begin{tabular}{lccc}
\hline & SPOT_ET & FUT_ET & Total \\
\hline Observações Brutas & 1.028 & 1.047 & 2.075 \\
(-) Exclusões & 12 & 31 & 43 \\
Amostra da Pesquisa & 1.016 & 1.016 & 2.032 \\
\hline
\end{tabular}

Legenda: SPOT_ET: Preços à vista do etanol hidratado base Paulínia/SP CEPEA/ESALQ; FUT_ET: Preços futuros do etanol CBOT.

Fonte: Resultados da Pesquisa (2016)

Após coletar estes dados, foi necessário realizar algumas exclusões. Em algumas datas, ocorreram negociações no mercado à vista e não ocorreu no mercado futuro, logo, foram excluídas, primeiramente, 12 observações. Posteriormente, foram desconsideradas 31 observações na série de mercado futuro, principalmente pelas datas relacionadas a feriados no Brasil e que não existem nos Estados Unidos.

O período foi escolhido devido a conveniência na disponibilização das cotações do preço futuro na CBOT e devido a indisponibilidade dos dados na bolsa de valores Brasileira. Após a coleta dos dados, e depois de procedimentos de exclusão de observações, excluindo-se feriados e demais dias em que não houve informação de preço da commodity no mercado spot ou futuro, a amostra final totalizou 2.032 observações diárias, correspondentes ao período de 03/10/2011 a 27/11/2015.

\subsection{Analise Exploratória dos Dados}

A primeira etapa deste estudo consistiu em realizar uma análise exploratória dos dados spot e futuro, com inicialmente sendo calculada a estatística descritiva dos preços e retornos analisados. Os retornos para a série foram obtidos a partir da seguinte relação:

$$
r=\ln \left(p_{t}\right)-\ln \left(p_{t-1}\right)
$$

Em que: $r$ é o retorno e $\ln \left(p_{t}\right)-\ln \left(p_{t-1}\right)$ a diferença entre os logaritmos dos preços. Algumas estatísticas descritivas básicas das séries são apresentadas na Tabela 2. 
Tabela 2 - Estatística descritiva: posição e dispersão

\begin{tabular}{c|c|c|c|c}
\hline & SPOT_ET & LN_SPOT_ET & FUT_ET & LN_FUT_ET \\
\hline Média & 1193,13 & 0,0002 & 2073,35 & $-0,0005$ \\
\hline Mediana & 1172,25 & 0 & 2149 & 0,001 \\
\hline Desvio-Padrão & 114,65 & 0,008 & 397,32 & 0,03 \\
\hline Mínimo & 1013 & $-0,03$ & 1308 & $-0,31$ \\
\hline Máximo & 1684,5 & 0,068 & 3517 & 0,098 \\
\hline $\begin{array}{c}\text { Coeficiente de } \\
\text { Variação }\end{array}$ & 0,1 & 27,95 & 0,19 & $-50,3$ \\
\hline
\end{tabular}

Legenda: SPOT_ET: Preços à vista do etanol hidratado base Paulínia/SP CEPEA/ESALQ; FUT_ET: Preços futuros do etanol CBOT; LN_SPOT_ET: Logaritmo dos preços à vista do etanol hidratado base Paulínia/SP CEPEA/ESALQ; LN_FUT_ET: Logaritmo dos preços futuros do etanol CBOT. Fonte: Resultados da Pesquisa (2016)

Para fins de ilustração, as Figuras 1 e 2 destacam o comportamento das observações diárias de preços spot do Etanol Hidratado base Paulínia (SP), os quais o CEPEA/ESALQ utiliza como referência para os preços nacionais, e no CBOT Denatured Fuel Ethanol Futures.

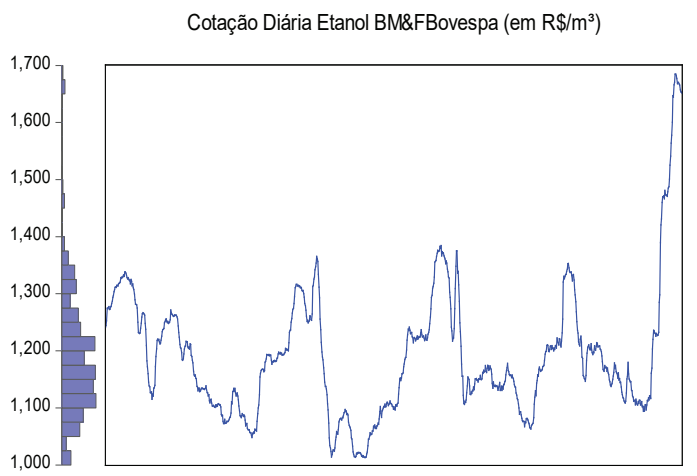

Figura 1 - Cotação diária Commodity Etanol spot, Período 03/10/2011 a 27/11/2015

Fonte: CEPEA/ESALQ (2015)

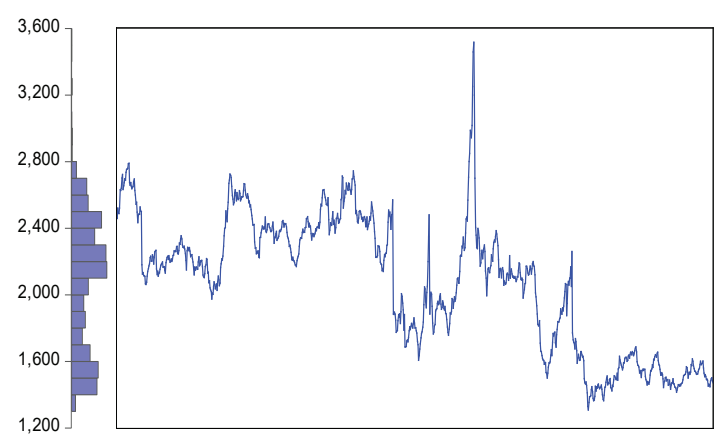

Figura 2 - Cotação diária Denatured Fuel Ethanol Futures, Período 03/10/2011 a 27/11/2015

Fonte: Chicago Mercantile Exchange Futures Data (2015)

De acordo com a série temporal acima, observa-se um período de alta no preço da commodity no spot, alcançando os $\mathrm{R} \$ 1.317 / \mathrm{m}^{3}$ e após este período, uma zona de

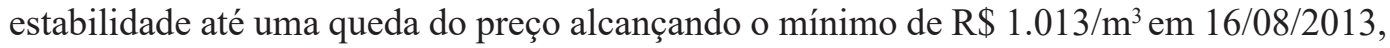
passando por um forte crescente a partir do final de setembro, alcançando o máximo de $\mathrm{R} \$ 1.684,50 / \mathrm{m}^{3}$ em 10/11/2015 $(\Delta+66 \%)$. Em relação às cotações no mercado futuro do etanol, a commodity passou por um período de alta volatilidade entre o final do segundo semestre de 2013 e o final de 2014, passando de um mínimo de US\$1.308/ $\mathrm{m}^{3}$ em 01/04/2014 até US\$ 3.517/ $\mathrm{m}^{3}$ em 16/01/2015. A Figura 3 destaca o comportamento dos retornos do preço spot do etanol hidratado no período analisado. 


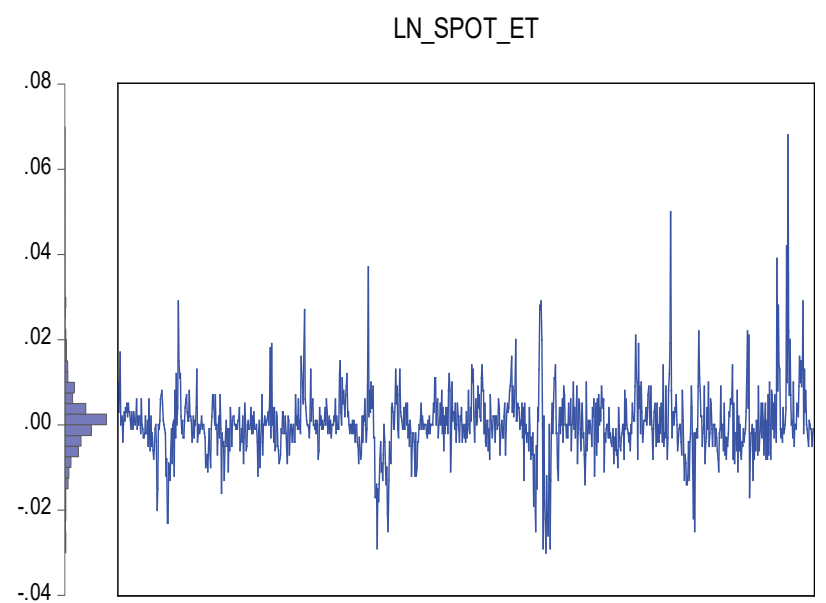

Figura 3 - Retornos diários Commodity Etanol spot, Período 03/10/2011 a 27/11/2015

Fonte: CEPEA/ESALQ (2015)

Consoante a Figura 3, através da qual se pode inferir sobre a volatilidade histórica e determinística do preço spot do Etanol Hidratado base Paulínia (SP), percebe-se que a série possui alguns retornos discrepantes, mas que permanecem entre -0,03 (maior queda, em 25/04/2015) e 0,07 (maior alta, em 30/09/2015). Em relação à volatilidade nos retornos diários da commodity no CBOT, em aproximados 15 meses, ocorreu de uma queda de 31\% no dia 06/09/2013, a uma alta de 10\% em 03/12/2014, conforme a Figura 4.

\section{LN_FUT_ET}

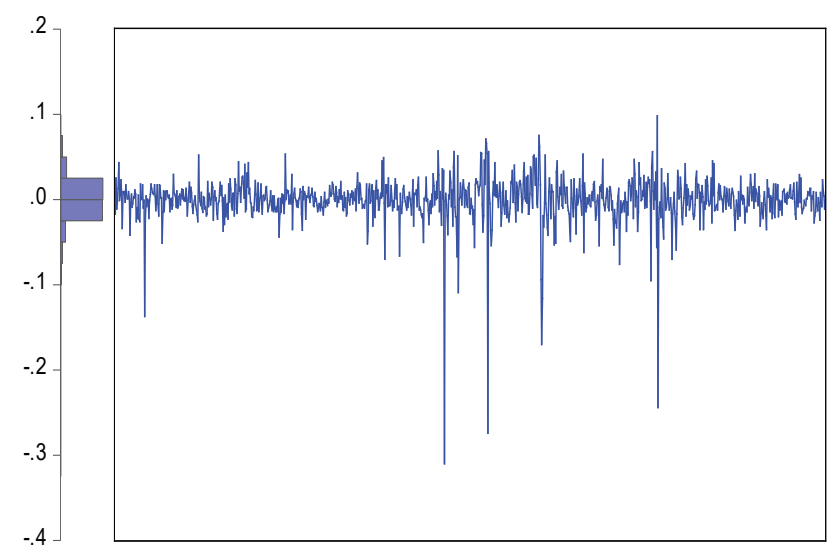

Figura 4 - Retornos diários Denatured Fuel Ethanol Futures, Período 03/10/2011 a 27/11/2015

Fonte: Chicago Mercantile Exchange Futures Data (2015)

Procedendo-se com a análise exploratória dos dados, em seguida, realizou-se a estatística de normalidade para a série de preços e retornos spot e futuro da commodity para o período de 03/10/2011 a 27/11/2015 a partir do teste de Jarque-Bera (JARQUE; BERA, 1987), com a finalidade de verificar se os resíduos destes apresentariam uma distribuição normal ou não. Consoante Gujarati (2012), o teste de normalidade JB é um teste assintótico 
(ou de amostra grande) baseados na assimetria e curtose das séries e utilizando o seguinte teste estatístico:

$$
J B=n\left[\frac{S^{2}}{6}+\frac{(K-3)^{2}}{24}\right]
$$

Em que: $n$ é o tamanho da amostra, $S$ é coeficiente de assimetria e $K$, o coeficiente de curtose. Para variáveis normalmente distribuídas, a assimetria (S) deve ser igual a 0 e a curtose $(\mathrm{K})$ igual a 3, esperando-se que o valor da estatística Jarque-Bera seja igual a zero. A Tabela 3 destaca os coeficientes de assimetria e curtose das séries de preços e retornos spot e futuro.

Tabela 3 - Estatística descritiva: formato

\begin{tabular}{ccccc}
\hline & SPOT_ET & LN_SPOT_ET & FUT_ET & LN_FUT_ET \\
\hline Assimetria & 1,488 & 1,125 & $-0,072$ & $-4,059$ \\
Curtose & 6,69 & 9,528 & 2,21 & 43,31 \\
\hline
\end{tabular}

Legenda: SPOT_ET: Preços à vista do etanol hidratado base Paulínia/SP CEPEA/ESALQ; FUT_ET: Preços futuros do etanol CBOT; LN_SPOT_ET: Logaritmo dos preços à vista do etanol hidratado base Paulínia/SP CEPEA/ESALQ; LN_FUT_ET: Logaritmo dos preços futuros do etanol CBOT.

Fonte: Resultados da Pesquisa (2016)

Considerados os parâmetros, e que sob a hipótese nula de normalidade, a estatística JB é distribuída como uma qui-quadrada com 2 graus de liberdade, em todas as séries indicou-se a rejeição da hipótese nula de normalidade, denotando-se que os retornos oscilam significativamente em função das informações que entraram no mercado (MONTE; AMIN; PENA, 2013). Para fins, a Tabela 4 e as Figuras 5 e 6 destacam os valores e o histograma dos preços e retornos spot e futuro no período analisado.

Tabela 4 - Jarque-Bera

\begin{tabular}{ccccc}
\hline & SPOT_ET & LN_SPOT_ET & FUT_ET & LN_FUT_ET \\
\hline $\begin{array}{c}\text { Jarque-Bera }\left(\mathrm{x}^{2}\right) \\
\begin{array}{c}\text { Jarque-Bera } \\
\text { (p-valor) }\end{array}\end{array}$ & 951,94 & 4078,2 & 27,22 & 71588,83 \\
\hline
\end{tabular}

Legenda: SPOT_ET: Preços à vista do etanol hidratado base Paulínia/SP CEPEA/ESALQ;

FUT_ET: Preços futuros do etanol CBOT; LN_SPOT_ET: Logaritmo dos preços à vista do etanol hidratado base Paulínia/SP CEPEA/ESALQ; LN_FUT_ET: Logaritmo dos preços futuros do etanol CBOT.

Fonte: Resultados da Pesquisa (2016) 


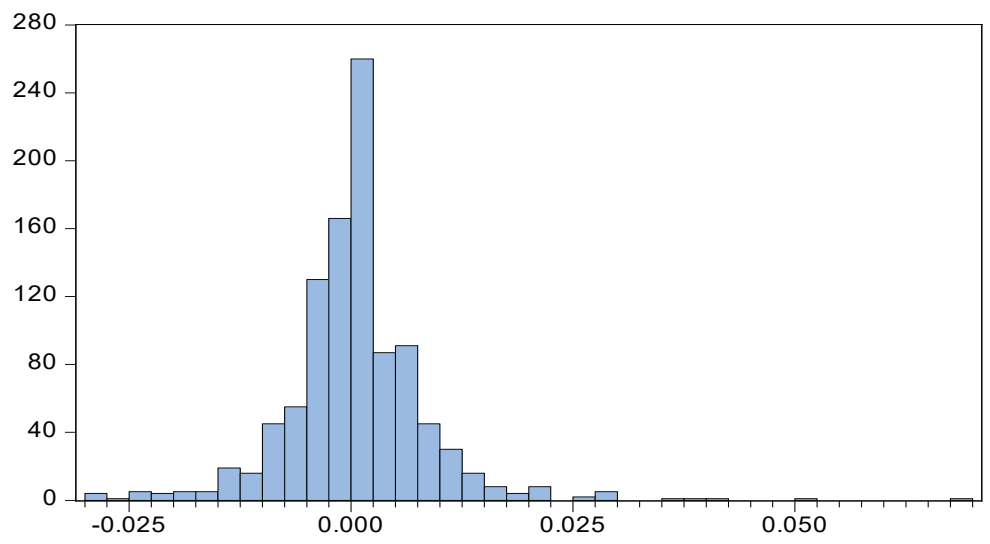

\begin{tabular}{ll} 
Series: LN_SPOT_ET \\
Sample 1 1016 \\
Observations & 1016 \\
Mean & 0.000284 \\
Median & 0.000000 \\
Maximum & 0.068000 \\
Minimum & -0.030000 \\
Std. Dev. & 0.007951 \\
Skewness & 1.126439 \\
Kurtosis & 12.55301 \\
& \\
Jarque-Bera & 4078.198 \\
Probability & 0.000000 \\
\hline
\end{tabular}

Figura 5 - Histograma dos retornos diários

Commodity Etanol spot, Período 03/10/2011 a 27/11/2015.

Fonte: CEPEA/ESALQ (2015)

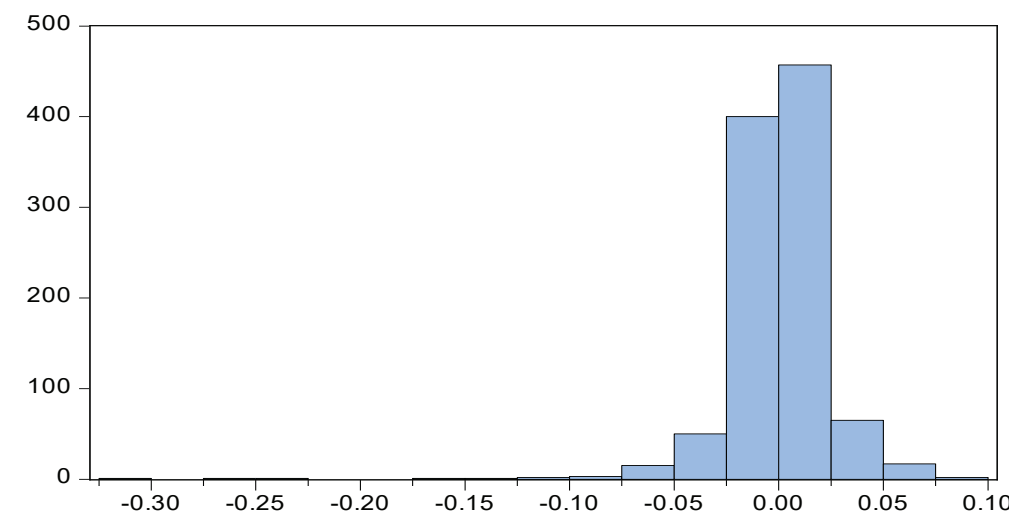

\begin{tabular}{|ll|}
\hline \multicolumn{2}{|l|}{ Series: LN_FUT_ET } \\
Sample 1 1016_- \\
Observations & 1016 \\
Mean & -0.000521 \\
Median & 0.001000 \\
Maximum & 0.098000 \\
Minimum & -0.310000 \\
Std. Dev. & 0.026193 \\
Skewness & -4.058696 \\
Kurtosis & 43.31355 \\
& \\
Jarque-Bera & 71588.83 \\
Probability & 0.000000 \\
\hline
\end{tabular}

Figura 6 - Histograma dos retornos diários Denatured Fuel

Ethanol Futures, Período 03/10/2011 a 27/11/2015.

Fonte: Chicago Mercantile Exchange Futures Data (2015)

Conforme as Figuras 5 e 6, o coeficiente de assimetria e de curtose indicam que a distribuição dos retornos spot é leptocúrtica e assimétrica à direita, uma vez que apresentam caudas mais pesadas (maiores) e um pico mais fino e alto devido ao excesso de curtose, comparadas à uma distribuição normal $(3,0)$. Além disso, considerados os resultados da estatística descritiva, observa-se que os quantis da direita estão mais afastados da mediana do que os da esquerda, ao contrário dos retornos diários no etanol futuro, que mantêm a curva leptocúrtica, porém, assimétrica à esquerda, de acordo com a Tabela 5. 
Tabela 5 - Percentis

\begin{tabular}{c|c|c|c|c|c|c|c|c|c}
\hline & P10 & P20 & P30 & P40 & P50 & P60 & P70 & P80 & P90 \\
\hline LN_SPOT_ET & $-0,008$ & $-0,004$ & $-0,002$ & 0,001 & 0,000 & 0,001 & 0,002 & 0,005 & 0,008 \\
LN_FUT_ET & $-0,020$ & $-0,012$ & $-0,008$ & $-0,003$ & 0,001 & 0,004 & 0,008 & 0,014 & 0,022 \\
\hline
\end{tabular}

Legenda: LN_SPOT_ET: Logaritmo dos preços à vista do etanol hidratado base Paulínia/SP CEPEA/ ESALQ; LN_FUT_ET: Logaritmo dos preços futuros do etanol CBOT.

Fonte: Resultados da Pesquisa (2016)

Em seguida, aplicaram-se os testes estatísticos Augmented Dickey Fuller (DICKEY; FULLER, 1979), Phillips-Perron (PHILLIPS; PERRON, 1988) e Kwiatkowski-Phillips-Schmidt-Shin (KWIATKOWSKI et al., 1992), que são aqueles convencionalmente indicados pela literatura para verificar a estacionariedade em séries temporais. Consoante Enders (2004), um processo estocástico é não estacionário se sua média varia com o tempo ou sua variância varia com o tempo, podendo ainda ocorrer ambas as situações. Os resultados destes testes para as séries de retornos spot e futuro do etanol encontram-se descritos na Tabela 6.

Tabela 6 - Testes de Raiz Unitária e Estacionariedade (p-valor)

\begin{tabular}{cccccccc}
\hline & \multicolumn{2}{c}{ ADF } & \multicolumn{2}{c}{ PP } & \multicolumn{2}{c}{ KPSS } \\
\hline & $\mathrm{p}$ & $p$-valor & Def. & $\mathrm{t}$-ajustado & $p$-valor & LM-t & $p$-valor \\
SPOT_ET & $-2,190929$ & 0,2099 & 1 & $-1,059353$ & 0,7334 & 0,280734 & 0,0000 \\
LN_SPOT_ET & $-10,99745$ & 0,0000 & 1 & $-21,87488$ & 0,0000 & 0,201474 & 0,2544 \\
FUT_ET & $-2,252612$ & 0,1880 & 1 & $-2,352113$ & 0,1559 & 2,743050 & 0,0000 \\
LN_FUT_ET & $-19,18854$ & 0,0000 & 1 & $-30,09518$ & 0,0000 & 0,025351 & 0,5265 \\
\hline
\end{tabular}

Legenda: SPOT_ET: Preços à vista do etanol hidratado base Paulínia/SP CEPEA/ESALQ; FUT_ET: Preços futuros do etanol CBOT; LN_SPOT_ET: Logaritmo dos preços à vista do etanol hidratado base Paulínia/SP CEPEA/ESALQ; LN_FUT_ET: Logaritmo dos preços futuros do etanol CBOT.

Fonte: Resultados da Pesquisa (2016)

Conforme os resultados dos testes ADF e PP apresentados na Tabela 6 verificou-se que, em primeira diferença I (1), os preços spot são estacionários, isto é, a média, variância e covariância são constantes. Logo, não existe raiz unitária, uma vez que a hipótese nula de presença de raiz unitária foi rejeitada ao nível de significância de $5 \%$ e consoante os seus valores críticos.

Em relação ao teste KPSS, os resultados apresentados indicaram que, quando logaritmizados em base neperiana, não se rejeitou a hipótese nula de que a série de retornos spot e futuro é estacionário. Assim, conclui-se que, em primeira diferença, existe estacionariedade para a série de preços à vista e futuro no período analisado. Com isso, uma vez que as séries apresentaram um comportamento de estacionariedade na primeira diferença, estas são classificadas como integradas de ordem I (1). 
No que tange à estacionariedade dos dados, de acordo com Gujarati (2012), um teste simples de estacionariedade também pode ser baseada na função de correlação (FAC) da série. A FAC, com defasagem $\mathrm{k}$, denotada por é definida por:

$$
\hat{p}_{k}=\frac{\hat{\gamma}_{k}}{\hat{\gamma}_{0}}=\frac{\text { covariancia com defasagem } k}{\text { variancia }}=\frac{\frac{\sum\left(Y_{t}-\bar{Y}\right)\left(Y_{t+k}-\bar{Y}\right)}{n}}{\frac{\sum\left(Y_{t}-\bar{Y}\right)^{2}}{n}}
$$

Em que: $n$ é o tamanho da amostra e $\bar{Y}$ é a média da amostra.

O correlograma das 1.016 observações de cada série (spot e futuro, 2.032 ao total) encontram-se nas Figuras 7 e 8, respectivamente, apresentados em até 20 defasagens, reduzidas pelo Critério de Informação de Akaike (AIC). O AIC define-se como:

$$
A I C=e^{2 k / n} \frac{\sum \hat{\mathrm{u}}_{i}^{2}}{n}=e^{2 k / n} \frac{\mathrm{SQR}}{n}
$$

Em que: $k$ é o número de regressores (incluindo o intercepto) e $n$ é o número de observações.

Com isso, o AIC impõe uma medida corretiva mais dura que $\mathrm{R}^{2}$ pelo acréscimo de regressores. Neste aspecto, ao comparamos dois ou mais modelos (modelos concorrentes, de acordo com Gujarati (2012), o com o valor mais baixo de AIC é preferido. No correlograma, a linha vertical no diagrama representa o eixo zero, as observações à direita são os valores positivos e à esquerda, os negativos. Para que exista um processo de ruído branco a autocorrelação estará próxima a zero em grande parte das defasagens, o que pode ser observados nas ilustrações.

De acordo com as Figuras 7 e 8, o padrão das autocorrelações total e parcial decaem de forma rápida para valores próximos de zero e se estabilizam já nas primeiras defasagens, destacando que a série de retornos spot pode ser considerada como estacionária. $\mathrm{O}$ correlograma é útil na verificação do tipo do modelo que fornece a melhor representação de uma série observada. Um correlograma, em que os valores de decaem para zero de forma relativamente lenta, indica uma possível não-estacionariedade da série, demandando uma diferenciação para tal.

\section{Resultados}

Após a análise exploratória da série dos preços e retornos, as volatilidades determinísticas por meio da estimação e identificação do modelo que consegue prever com maior acuracidade as variâncias condicionais foram calculadas. Para isso, foram utilizados os Critérios de Informação de Akaike (AIC) e de Schwarz (SC), destacando os modelos 


\begin{tabular}{|c|c|c|c|c|c|c|}
\hline Autocorrelation & Partial Correlation & & $A C$ & PAC & Q-Stat & Prob \\
\hline 吅 & 吅 & 1 & 0.436 & 0.436 & 193.58 & 0.000 \\
\hline שـ & $\square$ & 2 & 0.423 & 0.287 & 375.92 & 0.000 \\
\hline$\square$ & קו & 3 & 0.289 & 0.044 & 461.18 & 0.000 \\
\hline$\square$ & $\sqrt{1}$ & 4 & 0.224 & 0.004 & 512.40 & 0.000 \\
\hline طا & $d$ & 5 & 0.137 & -0.039 & 531.47 & 0.000 \\
\hline 川 & 다 & 6 & 0.024 & -0.112 & 532.06 & 0.000 \\
\hline 巾 & 中 & 7 & 0.036 & 0.022 & 533.41 & 0.000 \\
\hline$\|$ & $\|$ & 8 & -0.022 & -0.019 & 533.92 & 0.000 \\
\hline 1 & 川 & 9 & -0.035 & -0.020 & 535.19 & 0.000 \\
\hline 1 & 中 & 10 & -0.030 & 0.020 & 536.10 & 0.000 \\
\hline 1 & ما & 11 & -0.008 & 0.036 & 536.16 & 0.000 \\
\hline 1 & 1 & 12 & -0.004 & 0.005 & 536.18 & 0.000 \\
\hline 1 & 中 & 13 & 0.004 & 0.009 & 536.20 & 0.000 \\
\hline קו & קו & 14 & 0.080 & 0.093 & 542.89 & 0.000 \\
\hline i) & 1 & 15 & 0.026 & -0.045 & 543.60 & 0.000 \\
\hline 中 & 少 & 16 & 0.064 & 0.014 & 547.82 & 0.000 \\
\hline 巾 & 少 & 17 & 0.070 & 0.037 & 552.88 & 0.000 \\
\hline 1) & 1 & 18 & 0.033 & -0.048 & 554.01 & 0.000 \\
\hline 1 & 1) & 19 & 0.071 & 0.045 & 559.24 & 0.000 \\
\hline 巾 & 川 & 20 & 0.031 & 0.001 & 560.22 & 0.000 \\
\hline
\end{tabular}

Figura 7 - Correlograma Retornos Spot Fonte: CEPEA/ESALQ (2015)

\begin{tabular}{|c|c|c|c|c|c|c|}
\hline Autocorrelation & Partial Correlation & & $A C$ & PAC & Q-Stat & Prob \\
\hline 中少 & 巾 & 1 & 0.056 & 0.056 & 3.1783 & 0.075 \\
\hline 1 & 1 & 2 & 0.007 & 0.004 & 3.2345 & 0.198 \\
\hline 4 & 4 & 3 & -0.067 & -0.068 & 7.7683 & 0.051 \\
\hline 中 & i) & 4 & 0.024 & 0.031 & 8.3338 & 0.080 \\
\hline 1 & $\|$ & 5 & -0.008 & -0.010 & 8.3969 & 0.136 \\
\hline 川 & 川 & 6 & 0.019 & 0.015 & 8.7704 & 0.187 \\
\hline קו & קו & 7 & 0.043 & 0.045 & 10.638 & 0.155 \\
\hline 1 & 1 & 8 & 0.004 & -0.004 & 10.651 & 0.222 \\
\hline 나 & ㄴ. & 9 & -0.084 & -0.083 & 17.885 & 0.037 \\
\hline 1 & 川 & 10 & -0.005 & 0.010 & 17.907 & 0.057 \\
\hline d & 1 & 11 & -0.029 & -0.031 & 18.800 & 0.065 \\
\hline | & $\|$ & 12 & -0.003 & -0.010 & 18.807 & 0.093 \\
\hline 1 & $\|$ & 13 & -0.026 & -0.021 & 19.531 & 0.108 \\
\hline 1 & 4 & 14 & -0.048 & -0.053 & 21.864 & 0.081 \\
\hline 4 & 4 & 15 & -0.059 & -0.051 & 25.471 & 0.044 \\
\hline 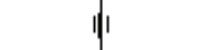 & | & 16 & -0.015 & -0.005 & 25.719 & 0.058 \\
\hline 1 & 1 & 17 & 0.008 & 0.005 & 25.779 & 0.079 \\
\hline 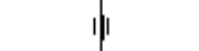 & 1 & 18 & 0.013 & 0.003 & 25.956 & 0.101 \\
\hline קו & קו & 19 & 0.049 & 0.052 & 28.496 & 0.074 \\
\hline קו & קו & 20 & 0.065 & 0.060 & 32.873 & 0.035 \\
\hline
\end{tabular}

Figura 8 - Correlograma Retornos Futuro

Fonte: Chicago Mercantile Exchange Futures Data (2015) 
com os menores valores para esses critérios. Primeiramente, buscou-se estimar os melhores modelos para a série de retornos spot do Etanol Hidratado base Paulínia (SP) ESALQ_no período de 03/10/2011 a 27/11/2015.

Consoante a Tabela 7, foram selecionados os modelos ARCH (3), GARCH $(1,1)$, TARCH $(1,1)$ e EGARCH $(2,2)$ para a estimação da série de retornos spot da commodity e os modelos ARCH (2), GARCH $(2,1)$, TARCH $(2,1)$ e EGARCH $(1,2)$ para a série de retornos futuro, os quais serão descritos a seguir.

Tabela 7 - Modelos ARCH Estimados: Retornos Spot e Futuro

\begin{tabular}{|c|c|c|c|c|}
\hline \multirow{2}{*}{ Modelos } & \multicolumn{2}{|c|}{ LN_SPOT_ET } & \multicolumn{2}{|c|}{ LN_FUT_ET } \\
\hline & Critério AIC & Critério SC & Critério AIC & Critério SC \\
\hline $\mathrm{ARCH}(1)$ & $-7,056182$ & $-7,046490$ & -4.806392 & -4.796700 \\
\hline $\mathrm{ARCH}(2)$ & $-7,154463$ & $-7,139925$ & -4.816193 & -4.801654 \\
\hline ARCH (3) & $-7,165275$ & $-7,145891$ & -4.813731 & -4.794346 \\
\hline $\mathrm{ARCH}(4)$ & $-7,163654$ & $-7,139424$ & -4.812199 & -4.787968 \\
\hline $\mathrm{ARCH}(5)$ & $-7,109647$ & $-7,080571$ & -4.818339 & -4.789262 \\
\hline $\begin{array}{c}\text { GARCH } \\
(1,1)\end{array}$ & $-7,161896$ & $-7,147358$ & -4.818344 & -4.803806 \\
\hline $\begin{array}{c}\text { GARCH } \\
(1,2)\end{array}$ & -7.160432 & -7.141048 & -4.819464 & -4.800079 \\
\hline $\begin{array}{c}\text { GARCH } \\
(2,1)\end{array}$ & -7.160107 & -7.140722 & -4.819581 & -4.800197 \\
\hline $\begin{array}{c}\text { GARCH } \\
(2,2)\end{array}$ & -7.157974 & -7.133744 & -4.817548 & -4.793318 \\
\hline $\begin{array}{c}\text { TARCH } \\
(1,1) \\
\end{array}$ & $-7,162827$ & $-7,143442$ & -4.868132 & -4.848748 \\
\hline $\begin{array}{c}\text { TARCH } \\
(1,2)\end{array}$ & -7.161351 & -7.137120 & -4.880532 & -4.856301 \\
\hline $\begin{array}{c}\text { TARCH } \\
(2,1)\end{array}$ & -7.160867 & -7.136636 & -4.883612 & -4.859382 \\
\hline $\begin{array}{c}\text { TARCH } \\
(2,2) \\
\end{array}$ & -7.162377 & -7.133301 & -4.879966 & -4.850889 \\
\hline $\begin{array}{c}\text { EGARCH } \\
(1,1)\end{array}$ & $-7,166350$ & $-7,146966$ & -4.862369 & -4.842984 \\
\hline $\begin{array}{c}\text { EGARCH } \\
(1,2)\end{array}$ & -7.172712 & -7.148481 & -4.889245 & -4.865014 \\
\hline $\begin{array}{c}\text { EGARCH } \\
(2,1)\end{array}$ & -7.170310 & -7.146080 & -4.869197 & -4.844966 \\
\hline $\begin{array}{c}\text { EGARCH } \\
(2,2)\end{array}$ & -7.174771 & -7.145694 & -4.888768 & -4.859692 \\
\hline
\end{tabular}

Legenda: LN_SPOT_ET: Logaritmo dos preços à vista do etanol hidratado base Paulínia/SP CEPEA/ ESALQ; LN_FUT_ET: Logaritmo dos preços futuros do etanol CBOT.

Fonte: Resultados da Pesquisa (2016) 
Considerando-se que o modelo com o melhor ajustamento para a série de retornos spot foi o ARCH (3), destaca-se a seguinte equação:

$$
\sigma_{t}=0,0000190+0,6090 \varepsilon_{t-1}^{2}+0,1385 \varepsilon_{t-2}^{2}+0,1164 \varepsilon_{t-3}^{2}
$$

Para a série de retornos futuros, o modelo com o melhor ajustamento foi o $\mathrm{ARCH}$ (2). Neste sentido, a equação resume-se como:

$$
\sigma_{t}=0.000255+0.675747 \varepsilon_{t-1}^{2}+0.152258 \varepsilon_{t-2}^{2}
$$

No caso dos modelos GARCH, em que a função de variância condicional inclui as variâncias passadas (), isto é, dos quadrados dos erros anteriores e também de sua própria variância (PONTES, 2013). Neste aspecto, uma vez que o melhor modelo para a série de retornos spot entre os modelos GARCH foi o $(1,1)$, chegou-se a seguinte equação:

$$
\sigma_{t}^{2}=0,0000145+0,601286 \varepsilon_{t-1}^{2}+0,274823 \sigma_{t-1}^{2}
$$

O somatório dos parâmetros $\alpha_{1}+\beta_{1}$ determina a persistência de choques na volatilidade do retorno em um curto prazo. De acordo com a equação, a dinâmica da volatilidade, isto é, o somatório dos coeficientes de reação e persistência ( $\Sigma \alpha_{1}=0,60+$ $\left.\beta_{1}=0,27\right)$ indica que os choques existentes possuem efeitos persistentes por longos períodos desta série - isto é, um choque de volatilidade tende a levar um maior tempo para se dissipar.

Em relação a série de retornos futuros, destacou-se um modelo $\operatorname{GARCH}(2,1)$ com a finalidade de mensurar a volatilidade da commodity no mercado americano. Com isso, chegou-se a seguinte forma funcional:

$$
\sigma_{t}^{2}=0,0000923+0.597763 \varepsilon_{t-1}^{2}-0.238684 \varepsilon_{t-2}^{2}+0.578388 \sigma_{t-1}^{2}
$$

De acordo com a equação acima, destaca-se que a reação da volatilidade captada pelo primeiro termo de erro $\left(0.597763 \varepsilon_{t-1}^{2}\right)$ é maior que no segundo momento $\left(-0.238684 \varepsilon_{t-2}^{2}\right)$. Além disso, destaca-se que a dinâmica da volatilidade foi de 0,937467 , demonstrando que os choques na volatilidade perduraram durante um longo período, fato esse justificado pela presença da distribuição leptocúrtica da série analisada. 
Para a série de retornos spot, o modelo EGARCH $(2,2)$ foi o que obteve o melhor ajustamento a partir dos AIC e SIC, chegando-se à seguinte equação:

$$
\begin{aligned}
\ln \left(\sigma_{t}^{2}\right)=-5 & , 137044+0,111662 \ln \left(\sigma_{t-1}^{2}\right)+0,462260 \ln \left(\sigma_{t-2}^{2}\right) \\
& +0,841180\left|\frac{\varepsilon_{t-1}}{\sigma_{t-1}}\right|+0,377854\left|\frac{\varepsilon_{t-2}}{\sigma_{t-2}}\right|+0,125856 \frac{\varepsilon_{t-1}}{\sigma_{t-1}}
\end{aligned}
$$

Os parâmetros ARCH $\alpha_{1}$ e $\alpha_{2}$ do modelo EGARCH $(2,2)$ medem o impacto marginal das informações sobre os retornos spot do etanol, cujos coeficientes foram $\alpha_{1}=$ 0,841180 e $\alpha_{2}=0,377854$. O termo $\ln \left(\sigma_{t-1}^{2}\right)$ ou seja, o $\beta_{i}$ do modelo GARCH, destaca a persistência da volatilidade, neste contexto, $\beta_{1}=0,111662$ e $\beta_{2}=0,462260$. Conforme visto, sendo apresentados por duas defasagens, é possível estabelecer comparações entre o primeiro e o segundo momento. Para a primeira defasagem, os coeficientes de reação e persistência da volatilidade $\left(\Sigma \alpha_{1}=0,84+\beta_{1}=0,11\right)$ indicam que os choques na volatilidade perduraram por muito tempo, a partir da série temporal analisada. Na segunda defasagem, o somatório $\left(\sum \alpha_{2}=0,38+\beta_{2}=0,46\right)$ destacou que a reação da volatilidade foi menor ao ingresso de informações ao mercado.

Em relação ao mercado futuro da commodity, o melhor modelo EGARCH foi o de ordem $(1,2)$, conforme a equação abaixo:

$$
\begin{gathered}
\ln \left(\sigma_{t}^{2}\right)=-0.917514+0.225101 \ln \left(\sigma_{t-1}^{2}\right)+0.691392 \ln \left(\sigma_{t-2}^{2}\right) \\
+0.396193\left|\frac{\varepsilon_{t-1}}{\sigma_{t-1}}\right|+0.198538 \frac{\varepsilon_{t-1}}{\sigma_{t-1}}
\end{gathered}
$$

Para este período, a dinâmica da série é do tipo persistente, uma vez que o somatório dos coeficientes de reação e persistência foi de 1,11, denotando uma série explosiva, mas não implicando necessariamente na não-estacionariedade da série analisada, e sim, a variância incondicional dos resíduos, conforme Nelson (1991). Com isso, seria necessário um maior espaço de tempo para que esse choque venha a dissiparse. Além disso, o $\gamma \neq 0$ confirma o efeito assimetria na série de retornos futuros do etanol, destacando que, os impactos resultantes dos choques positivos e negativos foram diferenciados na volatilidade, dentro do período analisado. 
Por fim, no caso do modelo TARCH, uma vez que o $(1,1)$ foi o que obteve um melhor ajustamento pelos AIC e SIC, chegou-se a seguinte equação:

$$
\sigma_{t}^{2}=0,0000144+0,6778 \varepsilon_{t-1}^{2}+0,282754 \sigma_{t-1}^{2}-0,185441 d_{t-1} \varepsilon_{t-1}^{2}
$$

A dinâmica da volatilidade para esta série através do modelo TARCH $(1,1)$, também medida pelo somatório dos coeficientes de reação e persistência da volatilidade ( $\left.\Sigma \alpha_{1}=0,68+\beta_{1}=0,28\right)$, destacam que os choques possuem um efeito de persistência por longos períodos na volatilidade desta série de retornos spot. Outra questão está relacionada aos efeitos assimetria e alavancagem da volatilidade nos retornos spot, que podem ser analisados sob a ótica do $\gamma$. Considerando-se que $\gamma=-0,185441 d_{t-1} \varepsilon_{t-1}^{2}$ $(\gamma \neq 0)$, os choques das notícias no mercado spot impactaram de forma diferenciada a volatilidade dos retornos da commodity. Além disso, o coeficiente negativo do $\gamma$ na equação indicou a não existência do efeito alavancagem nos retornos spot durante o período analisado.

No que se refere à série de retornos futuros do etanol, observou-se que os parâmetros estimados no modelo TARCH $(2,1)$ melhor sensibilizaram a dinâmica da volatilidade da série de retornos analisada, a partir da equação abaixo:

$$
\begin{gathered}
\sigma_{t}^{2}=0,0000429+0.536635 \varepsilon_{t-1}^{2}-0,188653 \varepsilon_{t-2}^{2}+0,773949 \sigma_{t-1}^{2} \\
-0,296143 d_{t-1} \varepsilon_{t-1}^{2}
\end{gathered}
$$

Conforme observado, o somatório dos coeficientes de reação e persistência da volatilidade a partir deste modelo é do tipo persistente, uma vez que $\Sigma \alpha_{1}=0,54+\alpha_{2}=$ $-0,19+\beta_{1}=0,77=1,12$, ora indicando que os choques persistem por um longo período na volatilidade da série de retornos analisados. Além disso, considerando-se o parâmetro $\gamma$ diferente de zero, destaca-se que os choques de notícias incorporadas ao mercado impactam na volatilidade destes retornos futuros do etanol. Outra questão na análise do TARCH $(2,1)$ é que se indicou a inexistência do efeito alavancagem para a série temporal. 


\section{CONSIDERAÇões FINAIS}

O etanol é uma fonte de energia natural, sustentável e renovável que pode ser produzido a partir de diversas matérias primas, como trigo, beterraba, milho e no caso do Brasil, em sua maioria, a partir da cana-de-açúcar, uma vez que a flexibilidade na produção do açúcar e do etanol na mesma unidade industrial se tornou uma variável importante para a maximização dos resultados das indústrias sucroenergéticas. Além disso, o biocombustível brasileiro possui resultados melhores quando comparados ao etanol produzido do milho, o que pode garantir ao etanol brasileiro o mercado norte-americano (NASTARI, 2014).

Outros fatos que justificam a utilização desta matéria-prima estão relacionados à tradição de quatro séculos no cultivo e utilização, a existência de solos adequados e não-escassos para o cultivo, além do próprio fornecimento do combustível necessário, o bagaço. Atrelam-se a esta dinâmica, uma alternativa para investimentos, o desenvolvimento econômico da atividade e a relação com o mercado internacional (LIEBERG, 2014).

Dentre os modelos analisados, destaca-se que os modelos EGARCH $(2,2)$ para o spot e EGARCH $(1,2)$ para o futuro são os que possuem o melhor ajustamento da série, considerado os Critérios de Informação de Akaike (AIC) e de Schwarz (SC).

Os parâmetros encontrados destacam que o impacto marginal das informações sobre os retornos spot e futuro do etanol são persistentes, assim como a sua volatilidade, uma vez que o somatório dos coeficientes de reação e persistência denotaram séries "explosivas", mas não implicando necessariamente na não-estacionariedade da série analisada, e sim, na variância incondicional dos resíduos. Estes fatos podem estar relacionados ao fato de que a formação de preços do etanol, tanto no Brasil, quanto nos Estados Unidos, além das dinâmicas anteriormente citadas, está essencialmente ligada à fatores de ordem micro e macroeconômica (CAMPOS, 2010).

Com isso, além dos determinantes da produção e da demanda do etanol, fundamentos e variáveis econômicas possuem estreita relação com a cotação desta commodity. Outro aspecto é que os agentes e analistas de mercado costumam questionar a importância destes fundamentos quando a volatilidade do mercado parece inexplicável.

\section{REFERÊNCIAS}

AGÊNCIA NACIONAL DO PETRÓLEO, GÁS NATURAL E BIOCOMBUSTÍVEIS - ANP. Anuário Estatístico Brasileiro do Petróleo, Gás Natural e Biocombustíveis 2015. Rio de Janeiro: ANP, 2015. Disponível em: http://goo.gl/CqcTzZ Acesso em: 23 mar. 2016.

BM\&FBOVESPA; COMITÊ DE VALORES MOBILIÁRIOS. Mercado de Derivativos no Brasil: Conceitos, Produtos e Operações. Rio de Janeiro: BM\&FBovespa - CVM, 2015. Disponível em: http://goo.gl/KKy0nNAcesso em 27 abr. 2016

BOLLERSLEV, T. Generalized Autorregressive Conditional Heteroskedasticity. Journal of Econometrics, v. 31, p. 303-27, 1986.

BUENO, R. de L. da S. Econometria de séries temporais. 2.ed. Cengage Learning: São Paulo, 2011. CAMPOS, S. K. Fundamentos econômicos da formação do preço internacional de açúcar e dos preços domésticos de açúcar e etanol. 145 p. Tese (Doutorado em Ciências, Área de Concentração: 
Economia Aplicada) - Universidade de São Paulo, Escola Superior de Agricultura "Luiz de Queiroz", Piracicaba, 2010.

COPELAND, T. E.; WESTON, J. F.; SHASTRI, K. Financial theory and corporate policy. 4 ed. Reading: Addison-Wesley, 2005.

DICKEY, D. A.; FULLER, W. A. Distribution of the estimators for autoregressive time series with a unit root. Journal of the American Statistical Association, 74, p. 427- 431, 1979.

ENGLE, R. F. Autorregressive conditional heterocedasticity with estimates of variance of UK inflation. Econometrica. v. 50, p. 987-1008, 1982.

GUJARATI, D. N. Econometria Básica. 5. ed. Porto Alegre: AMGH, 2012.

JARQUE, C. M.; BERA, A. K. “a test for normality of observations and regression residuals.” International Statistical Review, 1987. v. 55, p. 163-172.

KWIATKOWSKI, D.; PHILLIPS, P. C. B.; SCHMIDT, P.; SHIN, Y. Testing the null hypothesis of stationarity against the alternative of a unit root. Journal of Econometrics. n. 54, p. 159-178, 1992.

LIEBERG, V. A influência do estoque mundial de açúcar sobre o preço internacional dessa commodity. 2014. 87p. Dissertação (Mestrado em Economia e Gestão do Agronegócio) - Escola de Economia de São Paulo, Fundação Getúlio Vargas. São Paulo, 2014.

MONTE, L. F. O.; AMIM, M. M ; PENA, H. W. A. Análise da volatilidade do preço do cacau no mercado de futures de Nova York (CSCE): Uma aplicação dos modelos GARCH . Contribuciones a la Economía, v. 10, p. 17-34, 2013.

MORAES, M.; BACCHI, M. Etanol, do início às fases atuais de produção. Revista de Política Agrícola, ano 23, n. 4, p. 5-22, out./nov./dez. 2014.

NASTARI. P. Avaliação e perspectivas do setor sucroenergético. Brasília: Mapa, 2014. Disponível em: http://www.agricultura.gov.br/arq editor/file/camaras_setoriais/Acucar_e alcool/26RO/ App_Datagro_26RO_Alcool.pdf Acesso em 24 mar. 2016.

NELSON, D. B. Conditional heteroskedasticity in asset returns: a new approach. Econometrica, v. 59, n. 2, p. 347-370, 1991.

OLIVEIRA NETO, O. J.; GARCIA, F. G.; MACHADO, W. B. Hedge Completo versus Hedge Ótimo: qual a melhor estratégia de cobertura de risco para o mercado de boi gordo mineiro e paulista? In: Conferência em Gestão de Risco e Comercialização de Commodities, 2, 2012, São Paulo. Anais... São Paulo: BM\&FBovespa, 2012.

PHILLIPS, P. C. B.; PERRON, P. Testing for a unit root in time series regression. Biometrika, n. 75, p. $335346,1988$.

PONTES, T. T. e S. Precificação de opções sobre contratos futuros de boi gordo na BM\&BOVESPA: um estudo das volatilidades. Dissertação (Mestrado em Administração) - Programa de Pós-Graduação em Administração. Universidade Federal da Paraíba. João Pessoa, 2013.

SOUZA, W. A. da R. de. Avaliação de estratégias de gerenciamento de risco de preços de café do Brasil com o uso de mercados futuros. 2013. 143p. Tese (Livre-Docência) - Escola Superior de Agricultura "Luiz de Queiroz". Universidade de São Paulo. 2013.

TONIN, J. R.; PARRÉ, J. L.; TONIN, J. M. Análise de co-integração, efetividade e razão ótima de hedge para os mercados de etanol anidro brasileiro e norte-americano. In: Congresso Brasileiro de Economia e Sociologia Rural, 52, 2014, Goiânia. Anais... Goiânia: SOBER, 2014. p. 1-21. 
VIAN, C. E. F.; LIMA, R. A. S.; ABDO, M. D. Estudos de casos sobre as estratégias administrativas e operacionais utilizadas pelas usinas de açúcar e álcool da região de Ribeirão Preto, SP. A Economia em Revista. v. 15, n. 1, p. 5-20, 2007.

ZAKOIAN, J.M. Threshold heteroskedasticity models. Journal of Economic Dynamics and Control, v. 18, p. 931-955, 1994.

Submetido em: 7-4-2018

Aceito em: 4-5-2021 